\title{
Qual o risco de disfonia em trabalhadores que usam a voz em ambiente universitário?
}

\author{
What's the risk of dysphonia in workers who use their voice in a \\ university environment?
}

Bruna Gabriela Mechi da Silva ${ }^{1}$, Marcia Simões-Zenari ${ }^{1}$ (), Katia Nemr ${ }^{1}$

\section{RESUMO}

Objetivo: avaliar o risco de disfonia e a presença de alteração vocal em profissionais de diferentes categorias que atuam em ambiente universitário. Métodos: pesquisa transversal com uso do Protocolo de Rastreio de Risco de Disfonia para análise das variáveis gênero, idade, hidratação, tabagismo, categoria profissional e demanda vocal e para cálculo do escore final. A gravação da voz ocorreu no ambiente de trabalho, com uso do protocolo Consenso da Avaliação Perceptivo-Auditiva da Voz - CAPE-V. A análise perceptivo-auditiva foi feita por juízes. Os dados foram comparados entre os grupos alto/baixo risco de disfonia e presença/ausência de alteração vocal. Resultados: participaram 80 indivíduos, média de idade de 47,7 anos, $84 \%$ do gênero feminino. Observou-se alto risco de disfonia em $70 \%$ deles e alteração vocal em $34 \%$. Hidratação insuficiente e tabagismo foram frequentes. No grupo com risco elevado de disfonia e presença de alteração vocal destacaram-se os funcionários da área administrativa e, no grupo com menor risco e sem alteração, os funcionários da saúde. Idade mais avançada foi associada à presença de alteração vocal, independentemente dos grupos. Conclusão: a identificação do alto risco de disfonia e a relevante presença de alterações vocais em indivíduos de diferentes categorias profissionais que fazem parte do ambiente universitário sugerem a necessidade de propostas de ações preventivas e de promoção de saúde do trabalhador.

Palavras-chave: Distúrbios da voz; Disfonia; Fatores de risco; Saúde do trabalhador; Fonoaudiologia

\begin{abstract}
Purpose: To assess the risk of dysphonia and the presence of vocal alteration in professionals from different categories who work in a university environment. Methods: Cross-sectional research using the Dysphonia Risk Screening Protocol to analyze the variables sex, age, hydration, smoking, professional category and vocal demand and to calculate the final score. Voice recording took place in the work environment using the CAPE-V protocol. Judges performed the auditory-perceptual analysis. The data were compared between the groups with high / low risk of dysphonia and presence / absence of vocal disorders.

Results: 80 individuals participated, with an average age of 47.7 years, $84 \%$ female. There was a high risk of dysphonia in $70 \%$ and vocal alteration in $34 \%$. Insufficient hydration and smoking were frequent. In the group with a high risk of dysphonia and the presence of vocal disorders, the presence of employees in the administrative area stood out and in the group with lower risk and without changes, health employees. Older aged was associated with the presence of vocal disorders, regardless of the groups.

Conclusion: The identification of the high risk of dysphonia and the relevant presence of vocal alterations in individuals from different professional categories who are part of the university environment suggest the need for proposals for preventive actions and promotion of workers' health.
\end{abstract}

Keywords: Voice disorders; Dysphonia; Risk factors; Occupational health; Speech, Language and Hearing Sciences

\footnotetext{
Trabalho realizado no Departamento de Fonoaudiologia, Fisioterapia e Terapia Ocupacional, Faculdade de Medicina - FMUSP, Universidade de São Paulo USP - São Paulo (SP), Brasil.

${ }^{1}$ Laboratório de Investigação Fonoaudiológica em Voz, Departamento de Fisioterapia, Fonoaudiologia e Terapia Ocupacional, Faculdade de Medicina - FMUSP, Universidade de São Paulo - USP - São Paulo (SP), Brasil.

Conflito de interesses: Não.

Contribuição dos autores: $B G M S$ coleta, análise e interpretação dos dados e redação do trabalho; $M S Z$ análise e interpretação dos dados e revisão do trabalho; $K N$ concepção do estudo, análise e interpretação dos dados e revisão do trabalho.

Financiamento: Nada a declarar.

Autor correspondente: Marcia Simões-Zenari. E-mail: marciasz@usp.br

Recebido: Junho 17, 2020; Aceito: Fevereiro 15, 2021
} 


\section{INTRODUÇÃO}

Além de condições gerais de trabalho favoráveis para desenvolver suas atividades laborais adequadamente e com qualidade de vida, os indivíduos que utilizam a voz profissionalmente necessitam tanto da integridade das estruturas envolvidas na fonação, quanto de seu funcionamento, com boa qualidade vocal $^{(1)}$. A presença de distúrbios vocais pode comprometer a comunicação social e a ocupacional, além de produzir impactos negativos nos ouvintes, o que pode refletir desfavoravelmente na qualidade de vida do trabalhador ${ }^{(2,3)}$.

Além das alterações estruturais ou funcionais da laringe, os problemas de voz podem estar relacionados ao uso excessivo ou inadequado da voz, estresse, medicamentos, comorbidades, hábitos e estilo de vida do indivíduo. $\mathrm{O}$ avanço da idade também pode impactar negativamente. Fatores físicos, sociais, ambientais, organizacionais e psicológicos do trabalho também podem influenciar o aparecimento de alterações vocais ${ }^{(4)}$. Mulheres apresentam maior prevalência de disfonia e há categorias profissionais em que elas são maioria, como na educação básica e fundamental ${ }^{(5)}$.

A presença de alterações vocais em determinadas categorias profissionais pode sinalizar adoecimento coletivo determinado pelo desgaste da voz sob precárias condições ocupacionais. Assim, buscou-se por anos caracterizar o Distúrbio da Voz Relacionado ao Trabalho (DVRT), o que ocorreu em 2018. O DVRT é definido como qualquer forma de desvio vocal diretamente relacionado ao uso da voz durante a atividade profissional, que diminua, comprometa ou impeça a atuação e/ou comunicação do trabalhador, podendo, ou não, haver alteração orgânica da laringe $\mathrm{e}^{(6)}$.

No contexto da promoção da saúde de trabalhadores que utilizam profissionalmente a voz, é necessário o empenho dos profissionais da saúde e gestores para desenvolver ações que possibilitem rastrear o risco de disfonia e fatores associados, a fim de que se possam estabelecer estratégias focadas na perspectiva do nexo entre o trabalho e a voz, com a missão de que as condições e características do próprio trabalho sejam objeto de intervenções de promoção e proteção da voz desses trabalhadores ${ }^{(7)}$.

Há produção científica expressiva sobre a voz de professores e cantores decorrente da maior suscetibilidade para desenvolverem disfonia, porém, outras atividades profissionais podem apresentar alto risco para problemas de $\mathrm{voz}^{(8)}$. Profissionais como operadores de telemarketing, vendedores, religiosos, dentre outros, têm despertado o interesse dos especialistas ${ }^{(9-12)}$. Entretanto, outras categorias, como profissionais da saúde e aqueles que desenvolvem funções administrativas, por exemplo, não têm sido foco de estudos sobre o tema. Esses profissionais atuam em diferentes locais e têm maior ou menor demanda vocal, a depender de sua dinâmica de trabalho. Não foram localizados estudos dentre a bibliografia pesquisada que tenham se dedicado a investigar tais categorias em um mesmo ambiente profissional.

A classificação de Koufman e Isaacson ${ }^{(13)}$ propõe uma divisão em quatro níveis, de acordo com a demanda e a qualidade vocal requeridas para exercício da atividade profissional: o nível I, chamado de elite vocal, é composto por atores e cantores, que, além da alta demanda vocal, dependem de um expressivo refinamento da qualidade vocal; o nível II refere-se aos usuários profissionais da voz, como professores, palestrantes ou religiosos, e sua característica principal é que apenas uma alteração vocal de grau moderado acarretaria consequências; o nível III compreende os usuários não profissionais da voz (comerciantes, médicos, advogados) e, por fim, o nível IV inclui aqueles que não dependem da voz para sua atuação ${ }^{(13)}$.

Quando se atenta ao ambiente universitário, pode haver equivocada percepção de que somente os docentes de ensino superior necessitam de cuidados com a voz. Uma vez que as universidades abarcam diferentes cursos, de variadas especialidades, e necessitam de uma grande estrutura administrativa, existe uma enorme gama de profissionais que frequentam diariamente esse ambiente. Além de cursos das áreas de exatas, biológicas e humanas, existem os hospitais universitários, rádio e jornal, coros e até escolas de educação infantil, ensino fundamental e médio.

Na rotina da clínica de Fonoaudiologia da universidade em que a pesquisa foi desenvolvida e que resultou neste artigo, trabalhadores de diversas categorias profissionais procuram atendimento na área de voz, fato que despertou o interesse por não serem os profissionais da voz de elite (ou nível I) e, muitas vezes, nem de nível $\mathrm{II}^{(13)}$. Assim, ressalta-se que não somente os docentes de ensino superior que estão nesse ambiente podem apresentar elevado risco de disfonia, com prejuízos a sua atividade profissional e impacto na qualidade de vida. Por estar inserida nesse ambiente, é atribuição da Fonoaudiologia promover ações que melhorem esse cenário.

O objetivo deste estudo, portanto, foi avaliar o risco de disfonia e verificar a presença de alteração vocal em profissionais da voz que atuam em ambiente universitário, exceto docentes de ensino superior. Além disso, identificar hábitos de hidratação e tabagismo, demanda vocal, histórico de disfonia e condições do ambiente físico de trabalho.

\section{MÉTODO}

Trata-se de estudo transversal observacional aprovado por comitê de ética em pesquisa das instituições envolvidas (pareceres 3.342.404 e 2.827.746).

Nesta fase da pesquisa, foram incluídos os funcionários de uma universidade pública que estivessem cadastrados no Serviço Especializado em Engenharia de Segurança e Medicina do Trabalho (SESMT), cujas funções se enquadravam nos níveis de demanda vocal I, II ou II ${ }^{(13)}$. Os docentes de ensino superior não participaram desta fase por não serem cadastrados no SESMT e farão parte das próximas etapas de continuidade da pesquisa.

Integraram o estudo todos os profissionais que tiveram interesse, independente de idade, gênero, tempo de atividade na função, ou regime de trabalho na universidade, que participaram de todas as fases da pesquisa e que assinaram o Termo de Consentimento Livre e Esclarecido. Foram excluídos da amostra os profissionais que não completaram as fases da pesquisa e também os que apresentaram sintomas vocais relacionados a quadro de infecção de vias aéreas superiores, no momento da gravação da voz.

As funções exercidas pelos indivíduos foram distribuídas de forma a compor quatro categorias profissionais: administrativa (secretário, técnico de assuntos administrativos), educação (professor de educação infantil e ensino fundamental), saúde (enfermeiro, médico, fisioterapeuta, fonoaudiólogo, médico veterinário e psicólogo) e comunicação (jornalista, locutor, editor, regente de coral e agente cultural).

Os participantes receberam, em seu e-mail institucional disponível no cadastro do SESMT, o convite para participação, contendo informações sobre a pesquisa, instruções, o Termo de Consentimento Livre e Esclarecido e o link de acesso para 
preenchimento do Protocolo de Rastreio de Risco de Disfonia Geral (PRRD-G) ${ }^{(14)}$. À medida que os funcionários aderiam, eram enviados novos $e$-mails para aqueles que ainda não tinham respondido, fluxo usual na universidade, para participação em diferentes pesquisas científicas.

Os instrumentos e procedimentos adotados nesta fase do estudo foram:

- preenchimento do questionário eletrônico: o PRRD-G foi utilizado para calcular o risco de disfonia, por meio de seu escore que pode variar de 0 a 131 e, quanto maior a pontuação, maior o risco para disfonia; possibilita a classificação em alto ou baixo risco de disfonia, sendo o ponto de corte para risco elevado de 29,25 para mulheres, 22,75 para homens e 27,10 para idosos ${ }^{(14)}$. O PRRD-G possibilita o levantamento de diversos aspectos relacionados à presença de alterações vocais. Para este estudo, foram considerados os dados sobre hábitos relacionados ao uso da voz (hidratação e tabagismo), demanda vocal, histórico de disfonia e condições do ambiente de trabalho;

- gravação da voz: os participantes que preencheram o questionário foram contatados novamente, por e-mail, para agendamento da gravação da voz, a ser realizada no local de trabalho, em sala silenciosa; para as gravações, foi utilizado iPad (Apple Inc., EUA) com microfone acoplado (ShureMotiv MV88) e realizadas as tarefas vocais de emissão de vogais sustentadas, leitura de frases e fala espontânea, propostas no protocolo CAPE- $\mathrm{V}^{(15)}$.

A análise perceptivo-auditiva das vozes com o CAPE-V foi realizada pelas duas primeiras autoras, de modo que as avaliadoras não tiveram acesso à categoria profissional e outros dados que identificassem a voz analisada. Com base nas três diferentes tarefas vocais, foi atribuído o grau geral de desvio vocal e considerados com alteração vocal os profissionais com grau geral maior que $35,5^{(16)}$.

A partir da classificação do risco de disfonia, com o PRRD-G, e da presença de alteração vocal pela análise do grau geral de desvio vocal, com o CAPE-V, foram formados quatro grupos: baixo risco de disfonia e sem alteração vocal (A), baixo risco com alteração (B), alto risco sem alteração (C) e alto risco com alteração (D).

Os grupos foram comparados em relação às variáveis: gênero, idade, hidratação, tabagismo, categoria profissional e demanda vocal. A idade foi também analisada em relação às categorias profissionais. Por fim, todas as variáveis foram analisadas quanto à presença de alteração vocal, independente do risco de disfonia.

Realizou-se análise estatística descritiva geral e por grupo e aplicados os testes ANOVA One-Way e t-Student para as comparações em relação às variáveis numéricas. O teste Quiquadrado foi utilizado para analisar a associação entre as variáveis categóricas. Foi considerado nível de significância de 5\%.

\section{RESULTADOS}

Dos 1354 profissionais que fazem uso ocupacional da voz na universidade, 145 (11\%) devolveram os questionários preenchidos. Realizaram a gravação de voz 80 (55\%), o que representa $6 \%$ do total. A média de idade foi de 47,7 anos, com desvio padrão (DP) de 10,2. Participaram 67 (84\%) mulheres e $13(16 \%)$ homens.

Dentre os 80 trabalhadores, $30(37,5 \%)$ pertenciam à categoria administrativa (ADM), 22 (27,5\%) à área da saúde (SDE), 21 (26\%) eram profissionais da educação básica ou fundamental (EDU) e sete ( $9 \%$ ) da comunicação (COM).

Do total de participantes, $53(66 \%)$ indicaram tabagismo e 48 (60\%), hidratação insuficiente. A demanda vocal entre 2 horas e 1 minuto a 5 horas foi indicada por 22 (28\%) participantes; entre 5 horas e 1 minuto e 8 horas por $25(31 \%)$ e mais de 8 horas por $33(41 \%)$.

Em relação ao histórico de problemas vocais, 47 (59\%) trabalhadores relataram ter apresentado alteração vocal mais de uma vez e sete $(9 \%)$, pelo menos uma vez.

Sobre as condições do ambiente, 51 (64\%) profissionais relataram presença de poeira, $51(64 \%)$ apontaram ruído interno ou externo que interfere no uso da voz e 35 (44\%) indicaram trabalhar na presença de ar condicionado.

Quando distribuídos nos grupos, conforme risco e alteração vocal, caracterizaram-se em:

- Grupo A (baixo risco de disfonia e sem alteração vocal): $17(21 \%)$ trabalhadores (6 ADM, 6 SDE, 1 EDU e $3 \mathrm{COM})$, média de idade de 47 anos $(\mathrm{DP}=10,6), 16$ mulheres e um homem;

- Grupo B (baixo risco de disfonia e com alteração vocal): sete indivíduos (9\%) (4 ADM, 2 SDE, 1 EDU), média de idade de 52,7 anos $(\mathrm{DP}=12,9)$, seis mulheres e um homem;

- Grupo C (alto risco de disfonia e sem alteração vocal): 36 trabalhadores (45\%) (9 ADM, 10 EDU, 12 SDE e 4 COM), média de idade de 45,3 anos (DP = 10), 27 mulheres e nove homens;

- Grupo D (alto risco de disfonia e com alteração vocal): 20 trabalhadores (25\%) (11 ADM, 6 EDU, 3 SDE), média de idade de 51,1 anos $(\mathrm{DP}=8,4), 18$ mulheres e dois homens.

Foram observados $56(70 \%)$ profissionais com alto risco de disfonia e 27 (34\%) com alteração vocal.

Na comparação entre os grupos, em relação às variáveis selecionadas, não foi possível manter o grupo B devido ao reduzido número de participantes. Na comparação entre os três grupos (A, C e D), não se observou diferença em relação às variáveis estudadas: idade e gênero (Tabela 1),

Tabela 1. Comparação entre os grupos em relação à idade e gênero.

\begin{tabular}{lccccc}
\hline & Aspectos analisados & $\begin{array}{c}\text { Baixo risco e sem } \\
\text { alteração (A) }\end{array}$ & $\begin{array}{c}\text { Alto risco e sem } \\
\text { alteração (C) }\end{array}$ & $\begin{array}{c}\text { Alto risco e com } \\
\text { alteração (D) }\end{array}$ & $\begin{array}{c}\text { Teste de ANOVA } \\
\text { One-Way (p) }\end{array}$ \\
\hline Idade & Média & 47,1 & 45,3 & 51,1 & 0,110 \\
(anos) & Mediana & 46,0 & 44,0 & 52,0 & \\
& Desvio padrão & 10,6 & 10,0 & 8,4 & Teste Qui-quadrado (p) \\
Gênero & Feminino & $16(94,1)$ & $25(75,0)$ & $18(90,0)$ & 0,142 \\
$\mathrm{~N}(\%)$ & Masculino & $1(5,9)$ & $9(25,0)$ & $2(10,0)$ & \\
\hline
\end{tabular}

Legenda: $\mathrm{N}(\%)$ = número de sujeitos (percentual) 
hidratação, tabagismo, categoria profissional e demanda vocal (Tabela 2).

Devido à dispersão dos dados, não foi possível aplicar teste estatístico para comparar os grupos formados a partir do risco e da alteração vocal, em relação às categorias profissionais, pois algumas caselas ficaram com números abaixo de três. De maneira descritiva, ressalta-se que, no grupo com risco elevado de disfonia e presença de alteração vocal, $55 \%$ dos participantes eram da categoria ADM; com baixo risco e sem alteração vocal, $41,2 \%$ eram da SDE e com risco elevado e sem alteração vocal, $36,1 \%$ eram da EDU.

As médias de idade foram semelhantes nas diferentes categorias profissionais (Tabela 3 ). Ao se observar a mediana, constatou-se que, no grupo ADM, metade dos trabalhadores tinha mais de 52 anos e, no grupo SDE, mais de 51 anos (Tabela 3).

Considerando-se apenas a presença de disfonia, independentemente do risco de disfonia, foi observado que o grupo com alteração vocal apresentou média de idade mais elevada, em relação ao grupo sem alteração vocal (Tabela 4). Além disso, o grupo ADM ficou no limite da significância estatística, quanto ao número maior de profissionais com disfonia (Tabela 5). A ingesta hídrica considerada adequada pelo PRRD-G é de seis copos ou mais, correspondendo a, aproximadamente, 1,5 litros, ou mais, diariamente. Assim, observou-se que mais da metade da amostra apresentou hidratação insuficiente, bem como presença de tabagismo.

Tabela 2. Comparação entre os grupos em relação à hidratação, tabagismo, categoria profissional e demanda vocal.

\begin{tabular}{|c|c|c|c|c|c|c|c|c|c|c|}
\hline \multicolumn{2}{|c|}{ Aspectos analisados } & \multicolumn{2}{|c|}{$\begin{array}{c}\text { Baixo risco e sem } \\
\text { alteração (A) }\end{array}$} & \multicolumn{2}{|c|}{$\begin{array}{l}\text { Alto risco e sem } \\
\text { alteração (C) }\end{array}$} & \multicolumn{2}{|c|}{$\begin{array}{l}\text { Alto risco e com } \\
\text { alteração (D) }\end{array}$} & \multicolumn{2}{|c|}{ Total } & \multirow{2}{*}{$\begin{array}{l}\text { Teste Qui- } \\
\text { quadrado } \\
\text { (p) }\end{array}$} \\
\hline & & $\mathbf{N}$ & $\%$ & $\mathrm{~N}$ & $\%$ & $\mathrm{~N}$ & $\%$ & $\mathbf{N}$ & $\%$ & \\
\hline \multirow[t]{2}{*}{ Hidratação } & Adequada & 7 & 41,2 & 13 & 36,1 & 8 & 40,0 & 28 & 38,4 & 0,925 \\
\hline & Inadequada & 10 & 58,8 & 23 & 63,9 & 12 & 60,0 & 45 & 61,6 & \\
\hline \multirow[t]{2}{*}{ Tabagismo } & Ausência & 6 & 35,3 & 9 & 25,0 & 9 & 45,0 & 24 & 32,9 & 0,303 \\
\hline & Presença & 11 & 64,7 & 27 & 75,0 & 11 & 55,0 & 49 & 67,1 & \\
\hline \multirow[t]{3}{*}{ Demanda vocal } & 2 horas e 1 minuto a 5 horas & 6 & 35,3 & 10 & 27,8 & 5 & 25,0 & 21 & 28,8 & 0,217 \\
\hline & 5 horas e 1 minuto a 8 horas & 8 & 47,1 & 8 & 22,2 & 6 & 30,0 & 22 & 30,1 & \\
\hline & Mais de 8 horas & 3 & 17,6 & 18 & 50,0 & 9 & 45,0 & 30 & 41,1 & \\
\hline
\end{tabular}

Legenda: $\mathrm{N}$ = número de participantes; \% = percentual

Tabela 3. Comparação entre as categorias profissionais em relação à idade.

\begin{tabular}{lccccc}
\hline \multirow{2}{*}{ Idade (anos) } & \multicolumn{4}{c}{ Categorias profissionais } & \multicolumn{2}{c}{ Teste ANOVA } \\
\cline { 2 - 5 } & Administrativa & Comunicação & Educação & Saúde & 48,3 \\
Média & 49,2 & 44,1 & 46,5 & 51,0 & 0,618 \\
Mediana & 52,0 & 45,0 & 46,0 & 10,9 & \\
Desvio padrão & 10,2 & 7,7 & 10,6 & 22 & \\
$\mathrm{n}$ & 30 & 7 & 21 & 21 & \\
\hline
\end{tabular}

Legenda: $\mathrm{n}=$ número de sujeitos

Tabela 4. Comparação entre os grupos com e sem alteração vocal em relação à idade.

\begin{tabular}{lccc}
\hline \multirow{2}{*}{ Idade (anos) } & \multicolumn{2}{c}{ CAPE-V } & \multirow{2}{*}{ Teste t-Student ( $\mathbf{p})$} \\
\cline { 2 - 4 } & Sem alteração & Com alteração & $0,019^{*}$ \\
Média & 45,9 & 51,5 & \\
Mediana & 45,0 & 52,0 & 9,6 \\
Desvio padrão & 10,1 & 27 & \\
$\mathrm{n}$ & 53 & 27 & \\
\hline
\end{tabular}

*estatisticamente significativo

Legenda: CAPE-V = Consenso da Avaliação Perceptivo-Auditiva da Voz ; $n=$ número de sujeitos

Tabela 5. Comparação entre os grupos com e sem alteração vocal em relação ao gênero, hidratação, tabagismo, categoria profissional e demanda vocal.

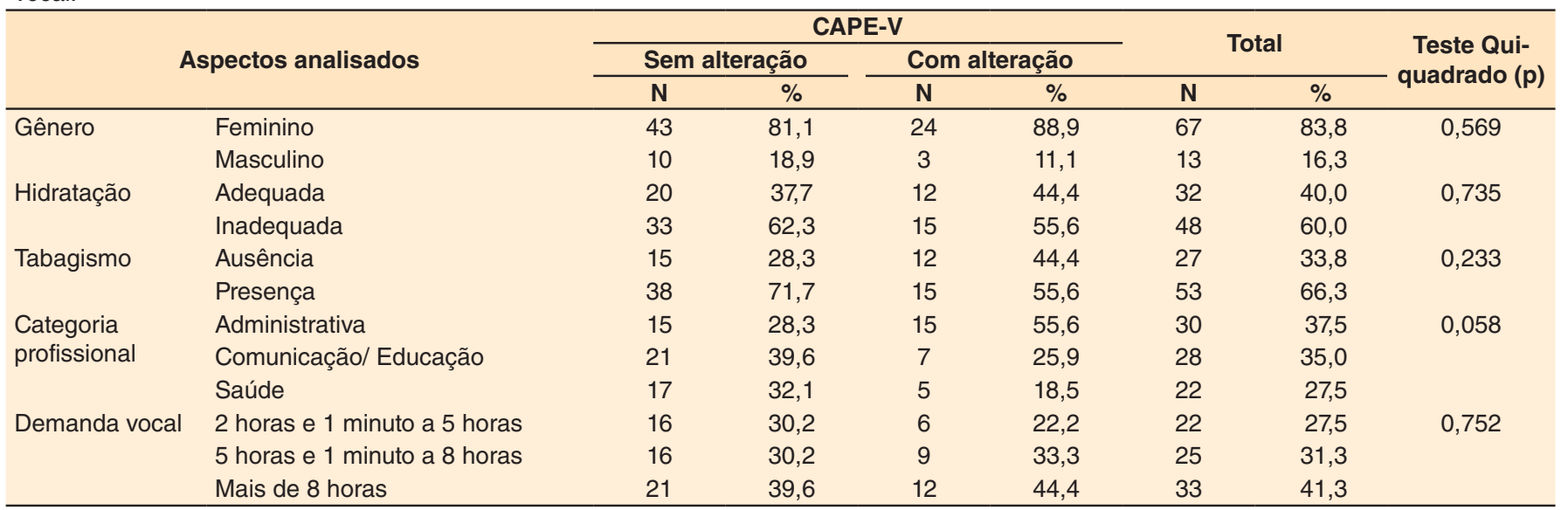

Legenda: CAPE-V = Consenso da Avaliação Perceptivo-Auditiva da Voz; $\mathrm{N}=$ número de sujeitos; \% = percentual 


\section{DISCUSSÃO}

Várias pessoas utilizam a voz profissionalmente e muitas acabam por desenvolver problemas vocais, sem que se deem conta que dependem da voz para exercer suas atividades laborais. É fundamental compreender as categorias profissionais nas quais essa questão é relevante e conhecer os fatores que estão levando esses trabalhadores ao adoecimento vocal.

Quando há uma referência ao uso da voz em ambiente universitário, naturalmente se pensa na demanda vocal do docente de ensino superior, mas esse universo abarca diferentes categorias profissionais com demandas vocais diversas. Nesse sentido, esta pesquisa incluiu categorias profissionais que não são corriqueiramente investigadas, porém, necessitam de atenção para tomada de decisões em saúde a partir de evidências científicas, para que ações preventivas e curativas possam ser desenvolvidas.

Um primeiro dado importante a ser considerado neste estudo foi a baixa adesão dos funcionários à proposta. A devolução dos questionários ficou muito aquém da média esperada, que seria em torno de $25 \%{ }^{(17)}$, e apenas metade dos participantes (aproximadamente) que preencheu o questionário, se dispôs a realizar a gravação da voz, mesmo com a proposta de que ocorresse no próprio local de trabalho. É possível que as pessoas mais expostas e/ou com mais alteração vocal sejam as que responderam ao questionário e participaram da gravação das vozes, fato que pode ser um viés do estudo, que não pôde ser controlado. Ações em que se promova discutir a questão do uso profissional da voz no âmbito universitário poderão contribuir com maior identificação desses profissionais com a temática, autopercepção em relação à voz e identificação de potenciais fatores de risco para disfonia.

Não houve diferença entre os grupos, em relação à idade, quando formados a partir da alteração vocal/risco de disfonia e quando compostos a partir da categoria profissional. Contudo, observou-se associação entre maior média de idade e presença de alteração vocal. Idade elevada pode favorecer a ocorrência de disfonia devido às mudanças fisiológicas causadas pelo envelhecimento ${ }^{(18)}$. Assim, ações específicas devem ser consideradas para os indivíduos com mais idade, de cada grupo. Além da idade e dos seus possíveis impactos na voz, as condições do ambiente de trabalho, os estados mentais e o estilo de vida também podem interferir na voz e devem ser aprofundados na investigação.

Assim como ocorreu nesta pesquisa, em geral, os homens participam menos de atividades relacionadas aos cuidados com a saúde por diversos motivos culturais e sociais e muito se tem refletido sobre a importância de estimular sua maior participação $^{(19,20)}$.

Risco elevado de disfonia e presença de alteração vocal entre aqueles que atuam na área administrativa chamam a atenção dos especialistas e indicam a necessidade de investigações que se voltem para as questões laborais dessa categoria profissional. Ressalta-se que o alto risco pode ser influenciado pelas condições de trabalho, como fatores ergonômicos, ruído, ar condicionado, alta carga mental e exigência laboral, questões relacionadas à organização e relações de trabalho, entre outros ${ }^{(21,22)}$, que precisam ser analisados futuramente.

Entre os participantes que eram professores de educação infantil e de ensino fundamental, o risco elevado de disfonia que foi observado era esperado, devido aos aspectos que envolvem a docência ${ }^{(5,23,24)}$. Também era esperada prevalência elevada de alteração vocal nesse grupo e, de fato, ficou acima do constatado no maior estudo epidemiológico realizado no Brasil ${ }^{(25)}$. O que eleva esse risco são fatores como condições desfavoráveis no ambiente de trabalho e estresse ${ }^{(23,24)}$, além de aspectos individuais, como comorbidades e tabagismo. Nesse cenário, deve-se considerar a existência de componentes organizacionais e emocionais do trabalho, como demanda excessiva, altas exigências, pouco controle e autonomia, que podem acarretar riscos aos trabalhadores, diminuição da qualidade de vida no trabalho e potencializar o risco de disfonia ${ }^{(26)}$. Em pesquisa anterior, professores de educação infantil e fundamental de escola particular de São Paulo apresentaram alto risco de disfonia com o mesmo instrumento utilizado neste estudo, independente de apresentarem, ou não, alteração vocal; a relação sinal-ruído mostrou-se inadequada em grande parte das salas de aula avaliadas, reforçando os possíveis impactos dos fatores ambientais ${ }^{(27)}$.

Os indícios de que o grupo de profissionais da saúde tem menor risco de disfonia podem estar associados à demanda vocal diferenciada, quando é necessário falar muito, porém em conversas e orientações mais individuais, nas quais os interlocutores estão mais próximos.

A hidratação insuficiente e o tabagismo elevado que foram observados são importantes fatores de risco para disfonia. Foi considerada insuficiente a ingestão de menos de 1,5 litros de água por dia. A literatura indica que o consumo de água deve variar de sete a 12 copos por dia, ou seja, mais de 1,5 litros e até 3,0 litros ${ }^{(28)}$. Nesse sentido, destaca-se que o consumo de água esteve abaixo do indicado, uma vez que mais da metade dos trabalhadores incluídos nesta pesquisa ingeria menos que 1,5 litros ao dia. A hidratação adequada contribui para o bom desempenho vocal e emissão confortável ${ }^{(28)}$, sendo preconizada ingestão de água em pequenas quantidades ao longo do dia, principalmente durante o uso profissional da voz.

Tabagismo elevado também foi encontrado em outras pesquisas sobre voz profissional e são bem estabelecidos os problemas vocais causados pelo fumo ${ }^{(29)}$. Estudo epidemiológico que analisou os distúrbios de voz em professores e não professores encontrou taxas de tabagismo 50\% mais elevadas nos brasileiros, em comparação aos americanos e que, tanto professores, quanto não professores brasileiros apresentaram taxas de tabagismo altas, ao redor de $80 \%{ }^{(25)}$. Considera-se importante o futuro detalhamento desses dados, investigando a quantidade de cigarros consumidos e tempo de tabagismo, tendo em vista os efeitos em longo prazo, e se ocorre associado a outros fatores, como consumo de bebidas alcoólicas, o que pode potencializar os efeitos vocais negativos.

Demanda vocal de mais de oito horas por dia foi relatada por expressivo número de participantes. Metade do grupo com risco elevado de disfonia e presença de alteração vocal apresentou essa alta demanda vocal. No grupo sem risco elevado e sem alteração, a menor parte relatou alta demanda. Esses dados fortalecem a ideia de que o distúrbio vocal não está atrelado apenas ao uso inadequado da voz, distanciando a culpabilização do trabalhador pelo agravo e endossando a necessidade de superação das condições mais desfavoráveis de trabalho ${ }^{(7)}$.

Além disso, as condições do ambiente físico indicam a presença de aspectos que interferem no uso da $\mathrm{voz}^{(5)}$ e o fato de muitos profissionais apresentarem histórico de disfonia tem que ser considerado, pois revela que esse cenário não se configurou agora. 
Para os profissionais com baixo risco de disfonia e com alteração vocal, que estiveram presentes em menor número, pressupõe-se que apresentem disfonias orgânicas. Portanto, faz-se necessário que, em estudos futuros, sejam considerados os aspectos de saúde geral e comorbidades para que a questão seja melhor elucidada. Esses profissionais e todos os demais que apresentaram alteração vocal serão encaminhados para o serviço de otorrinolaringologia do hospital de referência para diagnóstico laríngeo e também para avaliação fonoaudiológica, sendo então definidas as condutas. Os casos indicados serão notificados pela ficha de investigação de DVRT.

Para os participantes que não apresentaram alteração, mas foram identificados com alto risco para disfonia, a proposta é que oficinas de orientação sobre bem-estar vocal, autopercepção e aprimoramento vocal sejam realizadas. Para aqueles com risco baixo e que não apresentaram alteração vocal, estão em estudo propostas de elaboração de material de apoio que possa ser divulgado sem a necessidade de presença física.

As limitações da pesquisa se devem, principalmente, ao tamanho da amostra, ainda que os resultados tenham representado a realidade dessa população, que poderá ser beneficiada com as medidas de proteção.

Numa sociedade que valoriza políticas de saúde, os estudos epidemiológicos são relevantes; na área da voz, escassos e, por isso, desejáveis.

Ressalta-se que os achados do estudo alertam os especialistas em relação aos riscos elevados e ao alto índice de alteração vocal encontrados. Além disso, a baixa adesão deve nortear ações que motivem e conscientizem os profissionais da universidade sobre a relevância da voz para sua atividade profissional e medidas protetivas podem ser propostas aos gestores.

Com o estudo, pôde-se concluir que há risco elevado de disfonia entre os participantes, inclusive na ausência de alteração vocal, além de importante ocorrência de disfonia na amostra, como um todo.

Hidratação inadequada, tabagismo e alta demanda vocal se mostraram variáveis relevantes em todos os grupos estudados, assim como condições negativas do ambiente de trabalho, fatores a serem abordados nas ações que serão desenvolvidas. Questões específicas em relação aos funcionários da área administrativa também serão consideradas, uma vez que foi a categoria mais presente no grupo com risco elevado de disfonia e alteração vocal.

Isoladamente, as variáveis estudadas não diferenciaram os grupos, o que mostra a importância de que, em estudos futuros, sejam realizadas análises multivariadas e inseridos outros dados do PRRD. A continuidade da pesquisa é justificada pelo fato de $70 \%$ da amostra apresentarem risco elevado de disfonia e cerca de $30 \%$ terem alteração de voz, sendo necessário ampliar a compreensão sobre esse evento para delineamento e planejamento das ações. Destaca-se, ainda, que o cenário indica a necessidade de ações preventivas e de promoção da saúde a serem desenvolvidas com esses trabalhadores.

\section{CONCLUSÃO}

Foi encontrado elevado risco de disfonia e relevante presença de alteração vocal entre os profissionais de diferentes categorias que trabalham em ambiente universitário e que não atuam como docentes de ensino superior. Fatores como hidratação insuficiente, tabagismo elevado e condições negativas do ambiente físico foram observados.

\section{REFERÊNCIAS}

1. Cielo CA, Ribeiro VV, Hoffmann CF. Sintomas vocais de futuros profissionais da voz. Rev CEFAC. 2015;17(1):34-43. http://dx.doi. org/10.1590/1982-0216201517013.

2. Zambon F, Moreti F, Vargas AC, Behlau M. Efficiency and cutoff values of the Voice Activity and Participation Profile for nonteachers and teachers. Codas. 2015 Nov-Dez;27(6):598-603. http://dx.doi. org/10.1590/2317-1782/20152015028. PMid:26691625.

3. Ebersole B, Soni RS, Moran K, Lango M, Devarajan K, Jamal N. The influence of occupation on self-perceived vocal problems in patients with voice complaints. J Voice. 2017;32(6):673-80. http://dx.doi. org/10.1016/j.jvoice.2017.08.028. PMid:28967587.

4. Santana MC, Goulart BN, Chiari BM. Voice disorders in teachers: critical review on the worker's health surveillance practice. J Soc Bras Fonoaudiol. 2012;24(3):288-95. http://dx.doi.org/10.1590/S217964912012000300016 . PMid:23128179.

5. Byeon $\mathrm{H}$. The risk factors related to voice disorder in teachers: a systematic review and meta-analysis. Int J Environ Res Public Health. 2019;16(19):3675. http://dx.doi.org/10.3390/ijerph16193675. PMid:31574928.

6. Ferreira LP, Nakamura HY, Zampieri E, Constantini AC. Distúrbio de voz relacionado ao trabalho: proposta de uma ficha de notificação individual. Distúrb Comun. 2018;30(1):170-8. http://dx.doi. org/10.23925/2176-2724.2018v30i1p170-178.

7. Masson MLV, Ferrite S, Pereira LMS, Ferreira LP, Araújo TM. Em busca do reconhecimento do distúrbio de voz como doença relacionada ao trabalho: movimento histórico-político. Ciênc. saúde coletiva. 2019;24(3):805-16. http://dx.doi.org/10.1590/141381232018243.00502017 .

8. Mori MC, Francis DO, Song PC. Identifying occupations at risk for laryngeal disorders requiring specialty voice care. Otolaryngol Head Neck Surg. 2017;157(4):670-5. http://dx.doi.org/10.1177/0194599817726528. PMid:28895450.

9. Fuentes-López E, Fuente A, Contreras KV. Inadequate vocal hygiene habits associated with the presence of self-reported voice symptoms in telemarketers. Logoped Phoniatr Vocol. 2019;44(3):105-14. http:// dx.doi.org/10.1080/14015439.2017.1414302. PMid:29251526.

10. Natour YS, Darawsheh WB, Bashiti S, Wari M, Taha J, Odeh T. A study of VHI scores and acoustic features in street vendors as occupational voice users. J Commun Disord. 2018;71:11-21. http:// dx.doi.org/10.1016/j.jcomdis.2017.12.002. PMid:29223491.

11. Devadas U, Hegde M, Maruthy S. Prevalence of and risk factors for self-reported voice problems among hindu temple priests. J Voice. 2019;33(5):805.e1-12. http://dx.doi.org/10.1016/j.jvoice.2018.03.020. PMid:29748026.

12. Martins PN, Silverio KCA, Brasolotto AG. Vocal aspects of Brazilian evangelical pastors. J Voice. 2018;32(6):689-94. http://dx.doi. org/10.1016/j.jvoice.2017.08.014. PMid:28935208.

13. Koufman JA, Isaacson G. Voice disorders. Otolaryngol Clin North Am. 1991;24(5):989-98.

14. Nemr K, Simões-Zenari M, Duarte JMT, Lobrigate KE, Bagatini FA. Dysphonia risk screening protocol. Clinics (São Paulo). 2016;71(3):11427. http://dx.doi.org/10.6061/clinics/2016(03)01. PMid:27074171. 
15. Behlau M, Rocha B, Englert M, Madazio G. Validation of the Brazilian Portuguese CAPE-V Instrument - Br CAPE-V for Auditory-Perceptual Analysis. J Voice. 2020 Ago 15;S0892-1997(20)30257-5. http://dx.doi. org/10.1016/j.jvoice.2020.07.007. PMid:32811691.

16. Yamasaki R, Madazio G, Leão SHS, Padovani M, Azevedo R, Behlau M. Auditory-perceptual evaluation of normal and dysphonic voices using the voice deviation scale. J Voice. 2017;31(1):67-71. http:// dx.doi.org/10.1016/j.jvoice.2016.01.004. PMid:26873420.

17. Marconi MA, Lakatos EM. Fundamentos de metodologia científica.

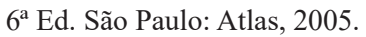

18. Mezzedimi C, Di Francesco M, Livi W, Spinosi MC, De Felice C. Objective evaluation of presbyphonia: spectroacoustic study on 142 patients with Praat. J Voice. 2017;31(2):257.e25-32. http://dx.doi. org/10.1016/j.jvoice.2016.05.022. PMid:27427181.

19. Teixeira DB. Atenção à saúde do homem: análise da sua resistência na procura dos serviços de saúde. Rev Cubana Enferm. [Internet]. 2016 [cited 2020 Jun 5];32(4). Available from: http://www.revenfermeria. sld.cu/index.php/enf/article/view/985/209

20. Arruda, G, Marcon, S. Reflexões sobre o cuidado ao homem adulto doente no âmbito familiar: uma perspectiva de gênero. Revista de Enfermagem da UFSM. 2016;6(2):298-306.

21. Fernandes C, Pereira A. Exposure to psychosocial risk factors in the context of work: a systematic review. Rev Saude Publica. 2016;50:24. http://dx.doi.org/10.1590/S1518-8787.2016050006129. PMid:27253900.

22. Almeida LMS, Dumith SC. Associação entre problemas vocais e tempo de trabalho em servidores públicos de uma Universidade Federal do sul do Brasil. Cad Saude Colet. 2018;26(3):249-54. http://dx.doi. org/10.1590/1414-462×201800030269.

23. Jardim R, Barreto SM, Assunção AA. Condições de trabalho, qualidade de vida e disfonia entre docentes. Cad Saude Publica. 2007;23(10):2439-61. http://dx.doi.org/10.1590/S0102-311X2007001000019. PMid:17891304.

24. Rissi V, Rozin S, Cecconelo WW. O impacto dos distúrbios de voz na qualidade de vida de docentes. Revista Profissão Docente. 2014;14(31):54-67.

25. Behlau M, Zambon F, Guerrieri AC, Roy N. Epidemiology of voice disorders in teachers and non-teachers in Brazil: prevalence and adverse effects. J Voice. 2012;26(5):665.e9-18. http://dx.doi.org/10.1016/j. jvoice.2011.09.010. PMid:22516316.

26. Giannini SPP, Latorre MDO, Ferreira LP. Distúrbio de Voz e estresse no trabalho docente: um estudo caso-controle. Cad Saude Publica. 2012;28(11):2115-24. http://dx.doi.org/10.1590/S0102311X2012001100011. PMid:23147953.

27. Silva BG, Chammas TV, Zenari MS, Moreira RR, Samelli AG, Nemr $\mathrm{K}$. Análise de possíveis fatores de interferência no uso da voz durante atividade docente. Rev Saude Publica. 2017;51:124. http://dx.doi. org/10.11606/S1518-8787.2017051000092. PMid:29236878.

28. Siqueira MA, Bastilha GR, Lima JPM, Cielo CA. Hidratação vocal em profissionais e futuros profissionais da voz. Rev CEFAC. 2016 Ago;18(4):908-14. http://dx.doi.org/10.1590/1982-0216201618417415.

29. Pinto AGL, Crespo AN, Mourão LF. Influência do tabagismo isolado e associado a aspectos multifatoriais nos parâmetros acústicos vocais. Braz J Otorhinolaryngol. 2014 Fev;80(1):60-7. https://doi. org/10.5935/1808-8694.20140013. 